\section{Check for updates}

Cite this: Soft Matter, 2017, 13,7300

Received 3rd September 2017, Accepted 21st September 2017

DOI: $10.1039 / \mathrm{c} 7 \mathrm{sm} 01773 a$

rsc.li/soft-matter-journal

\title{
Planar and 3D fibrous polyaniline-based materials for memristive elements
}

\author{
Yulia N. Malakhova, (D) $\dagger^{\mathrm{ab}}$ Alexei N. Korovin, $\dagger^{\mathrm{a}}$ Dmitry A. Lapkin, $\dagger^{\mathrm{a}}$ \\ Sergey N. Malakhov, ${ }^{a}$ Valentina V. Shcherban, ${ }^{b}$ Eugene B. Pichkur, ${ }^{a}$ \\ Sergey N. Yakunin, ${ }^{a}$ Vyacheslav A. Demin, ${ }^{a}$ Sergey N. Chvalun ${ }^{\mathrm{ab}}$ and \\ Victor Erokhin (iD *ac
}

\begin{abstract}
The memristive elements constructed using polymers - polyaniline (PANI) and polyethyleneoxide (PEO) could be assembled on planar thin films or on 3D fibrous materials. Planar conductive PANI-based materials were made using the Langmuir-Schaefer (LS) method, and the 3D materials - using the electrospinning method which is a scalable technique. We have analyzed the influence of PANI molar mass, natures of solvent and subphase on the crystalline structure, thickness and conductivity of planar LS films, and the influence of PANI molar mass and the PANI-PEO ratio on the morphological and structural characteristics of 3D fibrous materials.
\end{abstract}

\section{Introduction}

Memristive elements are non-volatile electronic components with prospective application as artificial synapses, ${ }^{1}$ components of perceptrons, ${ }^{2,3}$ and more complex neuromorphic networks. ${ }^{4}$ Their resistance depends on the applied voltage value and duration ${ }^{5}$ with a hysteresis loop in the current-voltage characteristics. Memristive elements could be based on semiconductor materials, ${ }^{6}$ which are able to change resistance reversibly as a result of oxygen vacancy migration. ${ }^{7}$

An organic memristive element based on a thin polyaniline (PANI) film was firstly described by Erokhin at al. ${ }^{8}$ Resistivity of such elements depends on the oxidation state of the thin PANI film via its electrochemical reaction with a silver chloride electrode, accompanied by ion migration through a separating polyethylene oxide (PEO) layer. ${ }^{8-13}$ The most reliable method of thin PANI layer preparation is transfer on the solid substrate of the Langmuir PANI layer formed on the water surface. Therefore, a deep understanding of the effect of Langmuir layer preparation conditions on its conductivity and crystallinity is required for the preparation of memristive devices with improved properties.

Compared with semiconductor materials, organic PANIbased memristive elements could be assembled not only by

\footnotetext{
${ }^{a}$ National Research Centre, Kurchatov Institute, 1, pl. Akademika Kurchatova, Moscow, 123182, Russia

${ }^{b}$ Moscow Technological University, Institute of Fine Chemical Technology, 86, Vernadskogo prosp., Moscow, 119571, Russia

${ }^{c}$ CNR-IMEM (National Research Council, Institute of Materials for Electronics and Magnetism) and University of Parma, Viale Usberti 7A, Parma, 42124, Italy.

E-mail: victor.erokhin@fis.unipr.it

$\dagger$ These authors contributed equally to this work.
}

laboratory-scale thin-film technologies but also by electrospinning-a wide-scale technique for the preparation of nonwoven 3D fibrous materials. ${ }^{14,15}$ Thereby the transition from an inorganic to fibrous architecture is perspective for creating stochastic neural networks.

In this work, we discuss the effect of memristive material preparation conditions (PANI molecular weight, the solvent and the Langmuir trough subphase compositions) on the structure formation of Langmuir PANI layers, performance of memristive thin-film elements as well as on the morphology and conductivity of electrospinned PANI-PEO nonwovens-perspective materials for memristive elements with a 3D microfiber architecture.

\section{Experimental}

\section{Materials}

Polyethylene oxide (PEO, $M_{\mathrm{w}}=1000000 \mathrm{~g} \mathrm{~mol}^{-1}$ and $8000000 \mathrm{~g} \mathrm{~mol}^{-1}$ ), a polyaniline emeraldine base with different molecular weights $\left(M_{\mathrm{w}}=10000 \mathrm{~g} \mathrm{~mol}^{-1}\right.$, PANI-10 and $M_{\mathrm{w}}=$ $100000 \mathrm{~g} \mathrm{~mol}^{-1}$, PANI-100) and lithium perchlorate (99\%) were purchased from Sigma-Aldrich, (1S)-(+)-10-camphorsulfonic acid (CSA, > 99\%) was obtained from Acros Organics. The solvents used for dissolving polymers (chloroform, toluene, $\mathrm{N}$-methyl-2-pyrrolidone) were of analytical grade and purchased from Component-Reaktiv. All chemicals except NMP were used as received without any further purification. NMP was distilled under vacuum prior to use.

\section{Methods}

Langmuir isotherm measurements. Langmuir isotherm measurement experiments were performed using Minitrough 
Extended (max area $558 \mathrm{~cm}^{2}$ ) and Minitrough (max area $243 \mathrm{~cm}^{2}$ ) Langmuir troughs (KSV, Finland) were used for the Langmuir layer formation. The compression speed of the barriers was $7.5 \mathrm{~cm}^{2} \min ^{-1}$.

Surface pressure was measured using the Wilhelmy method with a resolution of $0.1 \mathrm{mN} \mathrm{m}^{-1}$ using a rough platinum plate. The surface potential was measured by the vibrating electrode method using a SPOT sensor (KSV, Finland). The morphology of the PANI Langmuir layers directly on the water surface was visualized using a BAM300 Brewster microscope (KSV, Finland). Brewster microscopy images were geometrically corrected with allowance for observation at the Brewster angle $\left(53.1^{\circ}\right)$ and correspond to the interface region of $200 \times 200 \mu^{2}$. As a subphase we used demineralized water $\left(18.2 \mathrm{M} \Omega \mathrm{cm}\right.$ at $\left.25{ }^{\circ} \mathrm{C}\right)$ with pH 5.5 obtained using the Milli-Q Integral Water Purification System system (Millipore, USA) or the $\mathrm{HCl}$ or CSA aqueous solutions with $\mathrm{pH}$ 2.0. The Langmuir layer collapse was determined as a position of inflection at the surface pressure-area isotherms. The stability of the area of a compressed film before the collapse was confirmed by maintaining the constant values of the surface pressure.

Grazing incidence diffraction (GID). Grazing incidence diffraction (GID) experiments were performed at NRC "Kurchatov institute" at Langmuir beamline of a synchrotron radiation source (incident energy $13.6 \mathrm{keV}$ with an angle of incidence on the layer of $\mu=0.07^{\circ}$, i.e., $70 \%$ of the critical) and also at European Synchrotron Radiation Facility (ESRF) on the 10B beamline (incident energy $22.06 \mathrm{keV}$ with an angle of incidence on the layer of $\mu=0.045^{\circ}$, i.e., $80 \%$ of the critical). GID experiments were performed measuring the diffracted intensity as a function of the scattered angle using a point position sensitive detector or a linear position sensitive detector at NIC and ESRF, respectively. The reflected-scattered intensity was analyzed as a function of the in-plane $q_{x y}$ and the out of plane $q_{z}$ exchange momentum.

$\mathrm{X}$-Ray reflectivity measurements. X-Ray reflectivity measurements were performed at the ID10b beamline of the ESRF synchrotron facility, at the incident energy $\varepsilon=22.06 \mathrm{keV}$. Reflectivity curves were calculated by a custom-designed free-form approach for specular X-ray reflectivity ${ }^{16}$ adapted for modelling of thin polymeric films. This method will be discussed in detail in future publications.

Electrospinning. PEO spinning solutions were prepared by dissolving $100 \mathrm{mg}$ of PEO powder in $10 \mathrm{~mL}$ of chloroform and stirring using a magnetic stirrer for $24 \mathrm{~h}$. Spinning solutions of PANI-PEO were prepared in three steps. Firstly, required amounts of PANI and CSA (in the ratio of $1: 1.3$ ) were dissolved in $10 \mathrm{~mL}$ of chloroform and stirred using a magnetic stirrer for $24 \mathrm{~h}$. Then the solution was filtered using a glass filter (pore diameter $40-100 \mu \mathrm{m}$ ) to remove any particulate matter. Finally, $100 \mathrm{mg}$ of PEO powder was added to the solution and the mixture was stirred for another $24 \mathrm{~h}$.

Nonwoven materials were prepared using a laboratory electrospinning setup, which is schematically shown in Fig. 1. The distance between the needle and the collector was set at $30 \mathrm{~cm}$; applied voltage, generated by a Spellman SL130PN30 source,

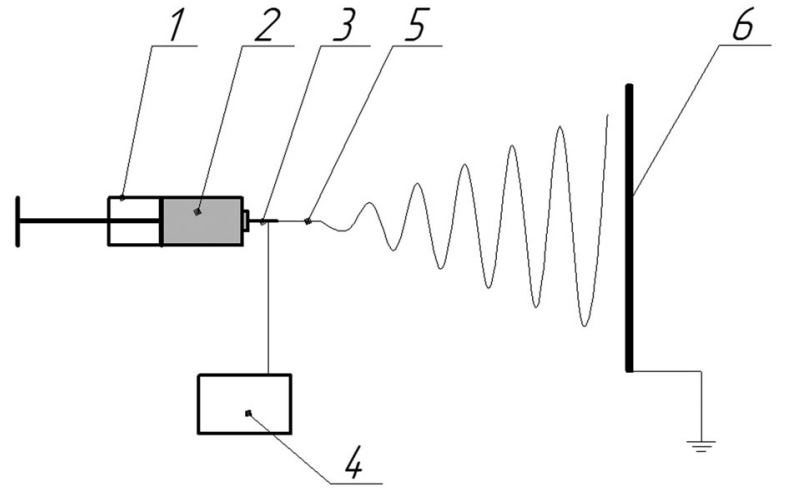

Fig. 1 Schematic diagram of electrospinning apparatus used for fiber production: 1 - pump, 2 - polymer solution, 3 - needle, 4 - high voltage source, 5 - primary jet, 6 - collecting electrode.

was $18 \mathrm{kV}$; the flow rate of the polymer solution, controlled with a precision pump, was $5 \mathrm{~mL} \mathrm{~h}^{-1}$. The morphology of the electrospun fibers was observed using a scanning electron microscope FEI Versa 3D at an accelerating voltage of $10 \mathrm{kV}$. Fiber diameters were measured using Image 1.49 software. The IR spectra were recorded using a Thermo Scientific Nicolet iS5 Fourier IR spectrometer and an iD5 ATR accessory in the range $550-4000 \mathrm{~cm}^{-1}$.

Polyaniline film preparation and characterization. For the preparation of the spreading solution, PANI was dissolved in NMP at a concentration of $1 \mathrm{~g} \mathrm{~L}^{-1}$ and filtered through a glass syringe filter with a pore size of $0.45 \mu \mathrm{m}$. The final solution of PANI was prepared by dilution of filtered solution with an NMP or an NMP : toluene 8:1 mixture to a concentration of $0.1 \mathrm{~g} \mathrm{~L}^{-1}$. Thereby as a spreading solvent, we used an NMP or an NMP: toluene $(9: 1)$ mixture.

Each PANI layer was transferred from an aqueous surface onto a solid substrate using the Langmuir-Schaefer method. Up to 80 consecutive layers were transferred on a single solid substrate. The surface morphology of solid substrates was investigated by atomic force microscopy (AFM) in tapping mode using a Multimode 8 microscope (Veeco, USA) with a controller Nanoscope V. The probe was a silicon cantilever RTESP (Bruker, USA) with a nominal force constant of $40 \mathrm{~N} \mathrm{~m}^{-1}$ and a resonance frequency of $300 \mathrm{kHz}$. The thickness of the coating was determined by the height difference between the film and the substrate surface after mechanical removal of the part of the coating.

As a substrate for conductivity measurements and memristive element assembly, we used glass slides with evaporated titanium electrodes with a $7 \mathrm{~mm}$ gap between them. Kapton stripes were placed on the substrate prior to polyaniline deposition so that after its removal the PANI film formed a $3 \mathrm{~mm}$ wide conductive channel between electrodes. The coatings deposited from subphases with pH 5.5 were thereafter converted to the conductive state by exposition in $\mathrm{HCl}$ vapor for $2 \mathrm{~min}$. Resistance was measured using a Keysight 34461A digital multimeter and then specific conductance was calculated with respect to geometry of the samples. 
Memristive element fabrication. The memristive element fabrication procedure is described elsewhere. ${ }^{11}$ On the conductive channel a drop of $\mathrm{LiClO}_{4}$ doped $(0.1 \mathrm{M})$ PEO $\left(M_{\mathrm{w}}=8 \mathrm{M}\right.$, $30 \mathrm{~g} \mathrm{~L}^{-1}$ water solution) stripe was placed to be used as a solid electrolyte. An Ag wire was inserted into the drop before it dried completely as a reference electrode and connected to one of the electrodes on the substrate. Characterization of the memristive elements was performed by means of a Keithley 4200-SCS Semiconductor Characterization System. Source and gate electrodes were grounded. Current-voltage curves of memristive elements were measured by scanning the applied source voltage from 0 to $+1.2 \mathrm{~V}$, then downwards from +1.2 to $-1.2 \mathrm{~V}$ and then back to $0 \mathrm{~V}$, as reported previously. ${ }^{8}$ The used voltage range is the widest window of both reversible electrochemical transitions of polyaniline and electrochemical stability of other components of the memristive device. Current values were measured $60 \mathrm{~s}$ after voltage application to ensure completion of the Red-Ox reaction in the active zone.

\section{Results and discussion}

\section{Planar memristive elements}

Spreading of polyaniline solution on the water surface with $\mathrm{pH} 5.5$ leads to the formation of Langmuir PANI layers in a nonconductive emeraldine base form. However, on the surface of the CSA or $\mathrm{HCl}$ aqueous solutions PANI is instantly protonated to a conducting emeraldine salt form. Therefore, we examined the effect of subphase composition as well as spreading solvent type and polyaniline molecular weight on PANI Langmuir layer formation. Typical surface pressure $(\pi)$ vs. relative surface area $(A)$ and the surface potential $(\Delta U) v s$. relative surface area compression isotherms of PANI are shown in Fig. 2. The shape of the $\pi-A$ isotherms and the $\pi$ values in the inflection points (when Langmuir layer collapsed) are consistent with the published data. ${ }^{17,18}$

An increase of PANI molecular weight from 10 to $100 \mathrm{~kg} \mathrm{~mol}^{-1}$ (PANI-10 and PANI-100, respectively) shows the stability increase of PANI Langmuir layers on the water surface by $5 \mathrm{mN} \mathrm{m}^{-1}$ (from 24.7 to $28.7-30.0 \mathrm{mN} \mathrm{m}^{-1}$ ) (Fig. 2a, curves 1 and 2) and a slight decrease of PANI Langmuir layers on the $\mathrm{HCl}$ aqueous solution surface by $2 \mathrm{mN} \mathrm{m}^{-1}$ (from 36.1 and $34.0 \mathrm{mN} \mathrm{m}^{-1}$ ) (Fig. 2b, curves 1 and 2). There is almost no change in the stability of Langmuir layers obtained from PANI solutions in the NMP: toluene mixture compared with solutions in NMP (Fig. 2a, curves 2 and 3). A change of subphase composition from water with $\mathrm{pH} 5.5$ to $\mathrm{HCl}$ solution with $\mathrm{pH} 2.0$ leads to the stability increase of the PANI Langmuir layer by $14 \mathrm{mN} \mathrm{m}^{-1}$ (from 24.7 to $42.9 \mathrm{mN} \mathrm{m}^{-1}$ ) for PANI-10 (Fig. 2a, curve 1 vs. Fig. 2b, curve 1) and by $10 \mathrm{mN} \mathrm{m}^{-1}$ (from 30 to $40.3 \mathrm{mN} \mathrm{m}^{-1}$ ) for PANI-100 (Fig. 2a, curve $3 v$ s. Fig. 2b, curve 2). However, a lower surface pressure stability value $\left(26.0 \mathrm{mN} \mathrm{m}^{-1}\right)$ was observed for Langmuir PANI layers on CSA aqueous solution compared with the water surface (Fig. 2b, curve 3 vs. Fig. 2a, curve 3).

A sharp increase of $\Delta U$ occurs in all experiments in the narrow area range before the $\pi$ increased and then $\Delta U$ monotonically increases during film compression followed by the

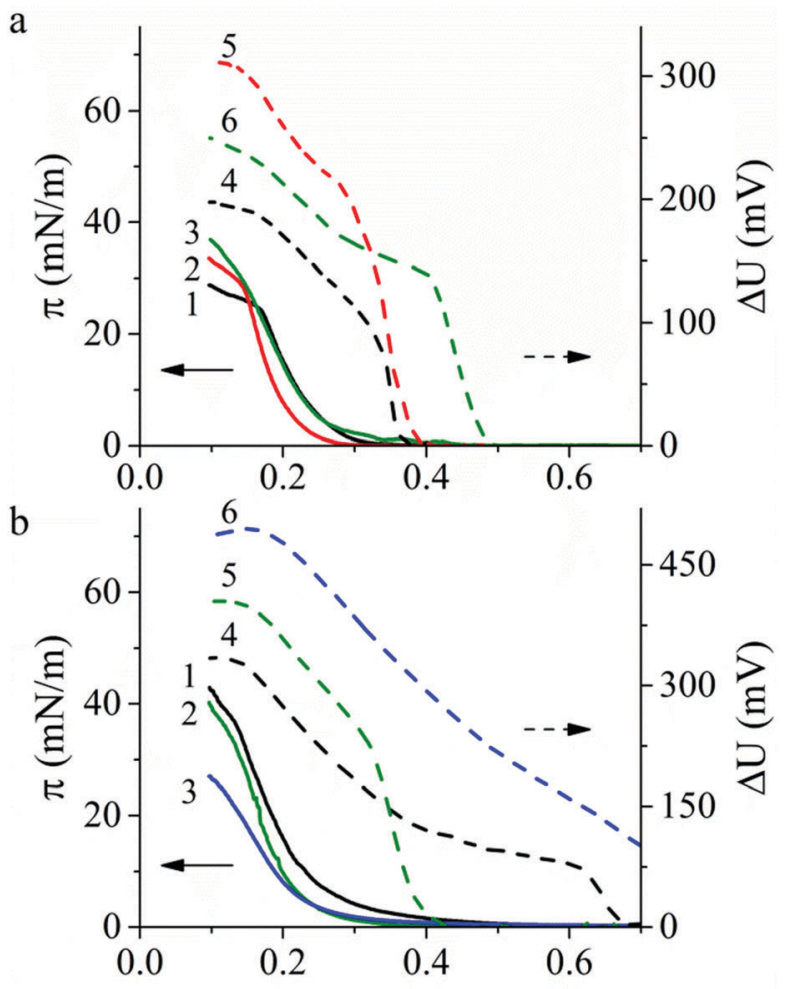

The proportion of the interfacial area

Fig. 2 Surface pressure $\pi(1-3)$ and surface potential $\Delta U(4-6)$ compression isotherms for Langmuir layers: (a) on the water surface, $\mathrm{pH}$ 5.5: PANI10 spread from NMP solution (1 and 4), PANI-100 spread from NMP solution (2 and 5), PANI-100 spread from a mixture of NMP and toluene solution ( 3 and 6); (b) on the $\mathrm{HCl}$ aqueous solutions surfaces, $\mathrm{pH}$ 2.0: PANI-10 spread from NMP solution (1 and 4), PANI-100 spread from mixture of NMP and toluene solution (2 and 5); on the CSA.

plateau region in the $\Delta U-A$ isotherm after the Langmuir film collapse. The maximum $\Delta U$ value depends on the subphase: 186-305 $\mathrm{mV}$ on the water surface and $330-490 \mathrm{mV}$ on the acid aqueous solution surface.

Brewster angle microscopy images of the surface layer of PANI 100k spread from PANI solution in a mixture of NMP and toluene on the CSA aqueous solution are shown in Fig. 3. After spreading the solution on the water surface and evaporation of

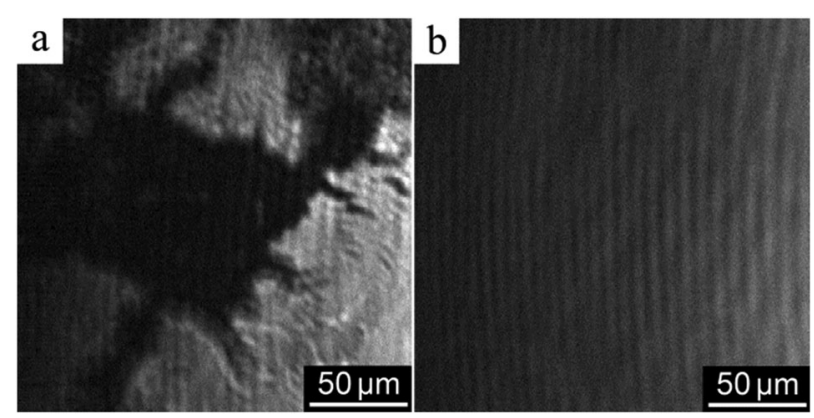

Fig. 3 Brewster angle microscopy images of Langmuir layers of PANI-100 spread from PANI solution in a mixture of NMP and toluene on the CSA subphase at surface pressure $0 \mathrm{mN} \mathrm{m}^{-1}$ (a) and $10 \mathrm{mN} \mathrm{m}^{-1}$ (b). 


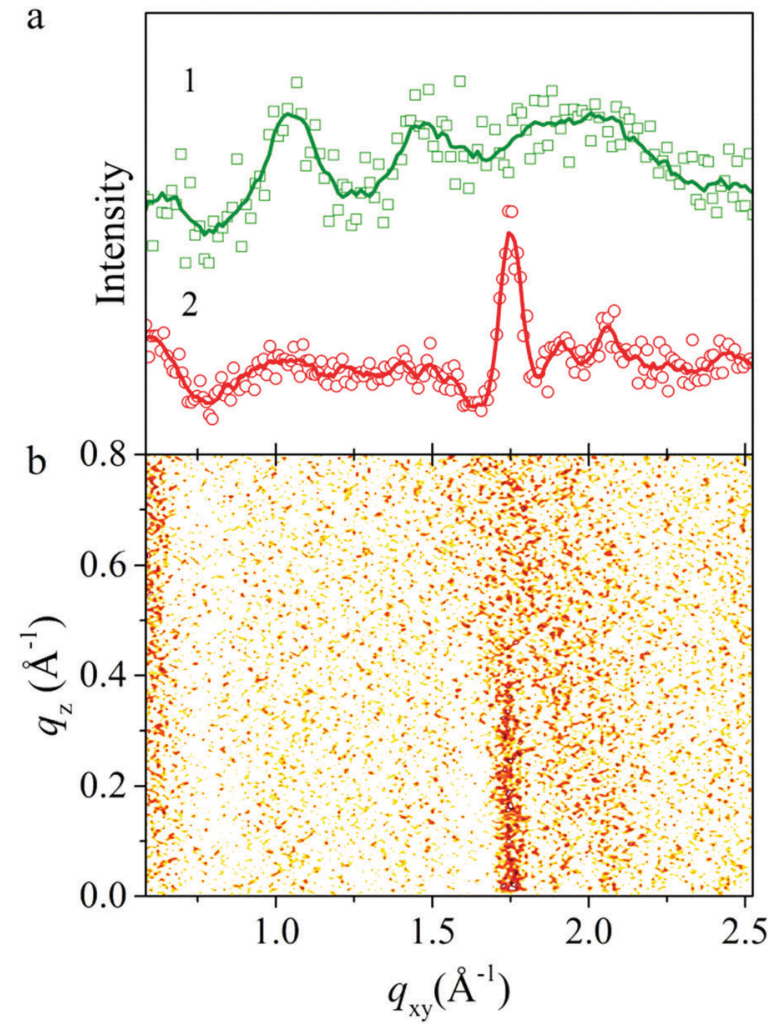

Fig. 4 (a) GIXD scattering intensity of Langmuir layer of PANI on various surfaces: $0.01 \mathrm{HCl}$ (curve 1), $0.01 \mathrm{M} \mathrm{CSA}$ (curve 2), (b) corresponding GID pattern for the film on the surface of $0.01 \mathrm{M} \mathrm{CSA}$.

the solvent, PANI forms islands of the condensed phase separated by the bare water surface (Fig. 3a). During compression, the film completely covers the interface at a relative interfacial area corresponding to the surface pressure increase (Fig. 3b). All the other PANI layers formed on either water or $\mathrm{HCl}$ aqueous solution surfaces behave similarly.

Langmuir layers were also studied by GID (Fig. 4). The diffraction pattern for the PANI Langmuir layer on the water surface with $\mathrm{pH} 5.5$ contains no diffraction peaks specific to PANI, which points to its amorphic structure. However the diffraction pattern for the Langmuir layer on the surface with of $0.01 \mathrm{M} \mathrm{HCl}$ (pH 2.0) contains reflexes that correspond to interplanar distances of 9.96, 6.10, 4.30 and $3.45 \AA$ (Fig. 4a, curve 1), characteristic of the crystallographic lattice of ES-I emeraldine salt. ${ }^{19}$

Compared with diffraction patterns of PANI discussed above, diffraction patterns of the PANI layer formed on the surface of $0.01 \mathrm{M}$ CSA solution at surface pressure in the range from 12 to $20 \mathrm{mN} \mathrm{m}^{-1}$ contain only a peak at $1.75 \AA^{-1}$, which corresponds to a lattice spacing of about $3.6 \AA$ (Fig. 4a, curve 2). This spacing is associated with the in-plane interchain stacking distance between phenyl rings. ${ }^{20}$ From FWHM of this peak following the Scherrer equation one can evaluate crystalline domain size that is equal to $7 \mathrm{~nm}$. Previously it was reported that such kinds of X-ray patterns for PANI-CSA films correspond to a highly conductive sample with metallic conductivity-temperature dependence and conductivity more than $10^{3} \mathrm{~S} \mathrm{~cm}^{-1} .^{21}$ The corresponding GID reciprocal space map scattering diagram of the PANI layer (Fig. $4 \mathrm{~b}$ ) shows a rod in the $q_{z}$ direction in crystalline domain size corresponding to the size of crystalline domains in the vertical direction of about $12 \AA$ and related to the lattice parameter $b$. Data on crystallinity of the samples are summarized in Table 1 .

The nature of solvent affected the mean thickness of the PANI monolayer. The thickness of the single LS layer was evaluated from the thickness of the films transferred to the glass substrate. The mean thickness of the PANI LS films spread from the NMP solution on the water surface is $3.7 \mathrm{~nm}$, whereas thickness of films spread from the solution in the mixture of NMP and toluene is $2.5 \mathrm{~nm}$. The thickness drastically increased to $6 \mathrm{~nm}$ when the PANI-10 solution was spread on the $\mathrm{HCl}$ acid aqueous solution surface. However, much thinner with a thickness of about $3.1 \mathrm{~nm}$ was formed on the surface of $0.01 \mathrm{M}$ CSA solution. X-ray reflectivity curves of this PANI layer (Fig. 5) indicated that variation of surface pressure in the range from 12 to $20 \mathrm{mN} \mathrm{m}^{-1}$ increased film roughness. However, the electronic distribution model points that the layer thickness at this pressure change increased only slightly from $21 \AA$ A to $24 \AA$ (Fig. 5, inset).

Data on conductivity of the PANI films are summarized in Table 1. The conductivity of PANI-10 LS films transferred from the water surface after doping in $\mathrm{HCl}$ vapors was $0.33 \mathrm{~S} \mathrm{~cm}^{-1}$. The increase of PANI molar mass to $100000 \mathrm{~g} \mathrm{~mol}^{-1}$ leads to the formation of Langmuir layers with amorphous structure, and the corresponding conductivity of the transferred film is about $2.6 \mathrm{~S} \mathrm{~cm}^{-1}$. PANI-100 spread from the PANI solution in a mixture of NMP and toluene on the water surface formed stable Langmuir layers with amorphous structure, and the corresponding conductivity for the deposited LS film is about $4.7 \mathrm{~S} \mathrm{~cm}^{-1}$. A change of subphase composition results in the formation of PANI-100 with a crystalline structure on the acidic aqueous solution surface Langmuir layers. The conductivity of the deposited LS films was 0.5 and $0.01 \mathrm{~S} \mathrm{~cm}^{-1}$ for Langmuir layers spread on $\mathrm{HCl}$ and CSA aqueous solution surfaces, respectively.

Table 1 Effect of solvent and subphase composition on the crystallinity of Langmuir PANI films and their corresponding conductivity after 60 sequential transfers onto the glass substrate

\begin{tabular}{lllllll}
\hline Entry & PANI molecular weight, $\mathrm{kg} \mathrm{mol}^{-1}$ & Solvent & Subphase & PANI crystallinity & Additional doping & Conductivity, S cm $^{-1}$ \\
\hline 1 & 10 & NMP & $\mathrm{H}_{2} \mathrm{O}, \mathrm{pH} \mathrm{5.5}$ & Amorphous & HCl vapor & 0.33 \\
2 & 100 & NMP & $\mathrm{H}_{2} \mathrm{O}, \mathrm{pH} 5.5$ & Amorphous & HCl vapor & 2.6 \\
3 & 100 & NMP : toluene & $\mathrm{H}_{2} \mathrm{O}, \mathrm{pH} 5.5$ & Amorphous & HCl vapor & 4.7 \\
4 & 100 & NMP: toluene & $\mathrm{H}_{2} \mathrm{O}+\mathrm{HCl}, \mathrm{pH} \mathrm{2.0}$ & Partially crystalline & - & 0.5 \\
5 & 100 & NMP : toluene & $\mathrm{H}_{2} \mathrm{O}+\mathrm{CSA}, \mathrm{pH} \mathrm{2.0}$ & Partially crystalline - &
\end{tabular}




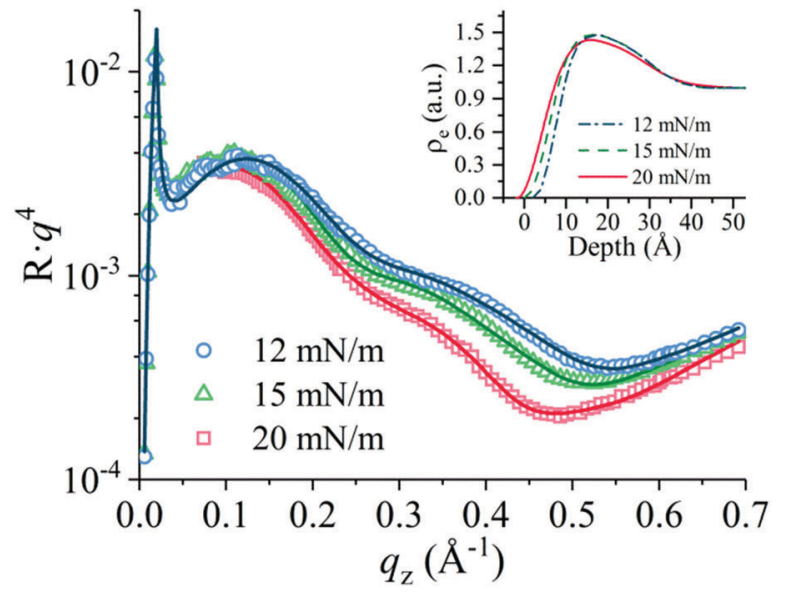

Fig. 5 XRR (data and fit) of a Langmuir film of PANI on the subphase with surface pressures of $12 \mathrm{mN} \mathrm{m}^{-1}$ (circles), $15 \mathrm{mN} \mathrm{m}^{-1}$ (triangles) and $20 \mathrm{mN} \mathrm{m}^{-1}$ (squares), scaled by the Fresnel reflectivity, vs. vertical momentum $q_{z}$; the corresponding electronic distribution model in the inset.

For the memristive element fabrication we used samples with the highest conductivity i.e. the PANI-100 film produced by 60 sequential transfers of Langmuir layers formed by spreading PANI solution in a mixture of NMP and toluene on a water surface and subsequent protonation with $\mathrm{HCl}$ vapors (Table 1, entry 3). In this study the technology of thin-film planar memristive element assembly was similar to that described in the previous papers. $^{8-12,22} I-V$ curves for the elements obtained at the day of preparation and 6 days later are represented in Fig. 6.

Curves of total current show hysteretic behavior with resistive switching. Conductance increases when the applied voltage is above $+0.4 \mathrm{~V}$ and decreases when it is below $+0.1 \mathrm{~V}$ due to oxidation and reduction of the PANI layer, respectively (Fig. 6a). The corresponding peaks in the ionic current through the gate electrode on these voltage values (Fig. 6b) are in good agreement with the RedOx potential $v s$. silver chloride electrode equal to $+0.3 \mathrm{~V}$. The sharp increase of ionic current at voltages higher than $+1 \mathrm{~V}$ can be attributed to the electrolysis of water in the electrolyte layer. Since the Red-Ox state and thus the conductance of the PANI channel remains almost unchanged under an applied voltage of $+0.3 \mathrm{~V}$, this voltage value was assigned as a test voltage. The conductance ratio between high-conductive and low-conductive states of the element at the test voltage measured as prepared was about 50 and measured after 6 days decreased to 9 accompanied by a general drop in the total conductance due to $\mathrm{HCl}$ vaporization. Furthermore, a change in the peak positions of the ionic current indicates a slowing of the reactions. A possible reason for this observation may be a slowing down of $\mathrm{Li}^{+}$diffusion due to evaporation of water from the PEO layer. Reducing the peak at voltages above $+1 \mathrm{~V}$ is another possible indication of a decrease in the amount of water in the electrolyte. Resistive switching kinetics of the memristive elements is fully consistent with the literature ${ }^{9,13}$ and so omitted.

\section{Nonwoven memristive elements}

Nonwoven materials from PEO and PANI-PEO mixtures were produced by electrospinning to obtain $3 \mathrm{D}$ memristive elements.
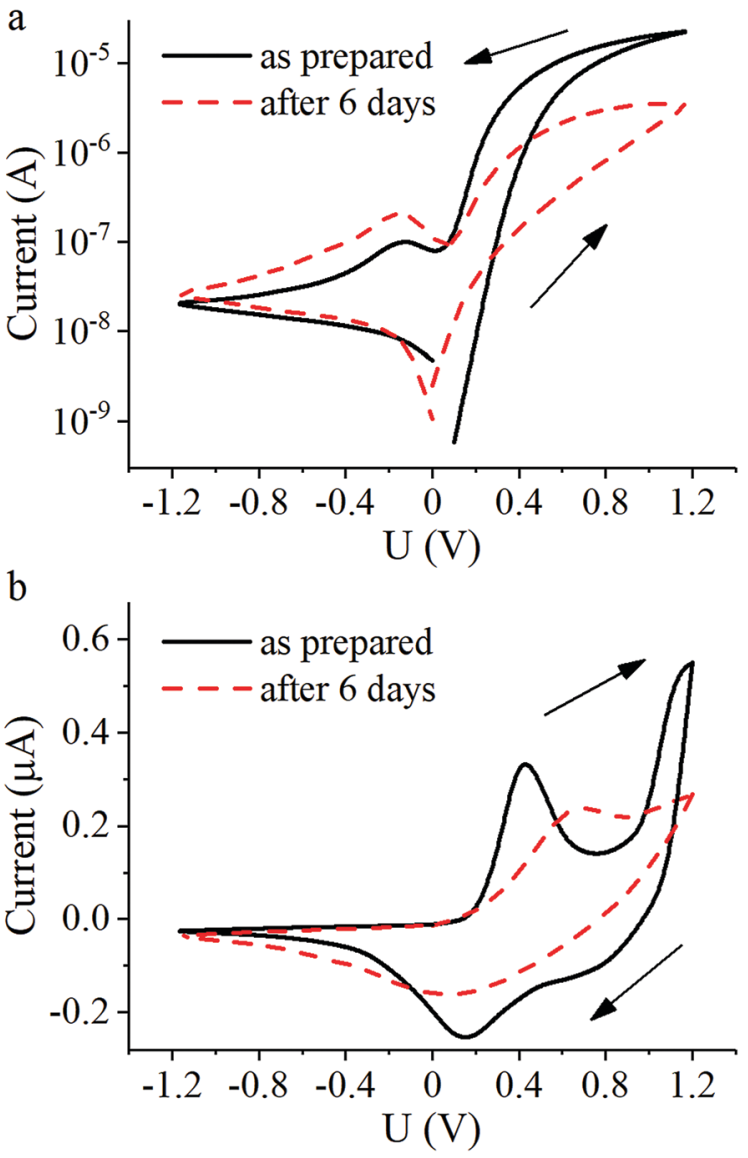

Fig. $6 \quad I-V$ curves of planar PANI-based memristive element: (a) total current and (b) ionic current.

Fig. 7 shows a micrograph of the obtained fibers, and a histogram of their distribution by diameter.

As can be seen from the micrographs, the fibers in the nonwoven material, produced from the PEO solution, have a smooth surface but a non-circular shape. The addition of PANI to the solution leads to the production of fibers that are more

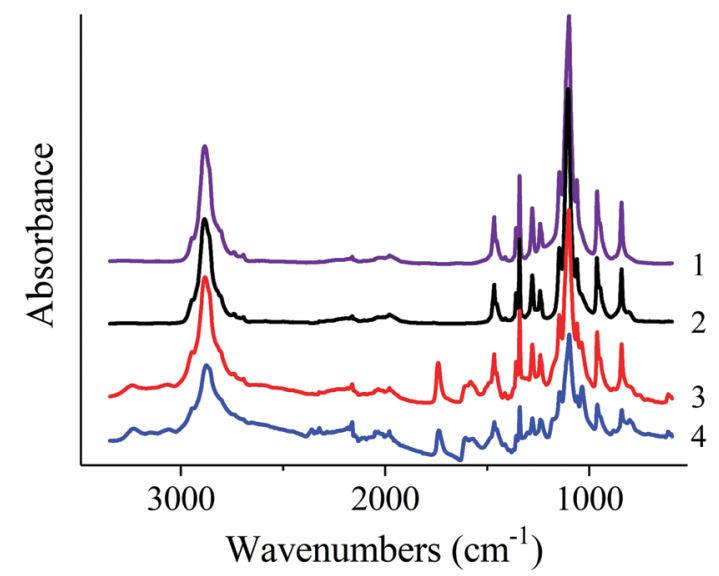

Fig. 7 FTIR spectra of the native PEO (1); PEO nonwoven (2); PEO + PANI-10 nonwoven (3); PEO + PANI-100 nonwoven (4). 

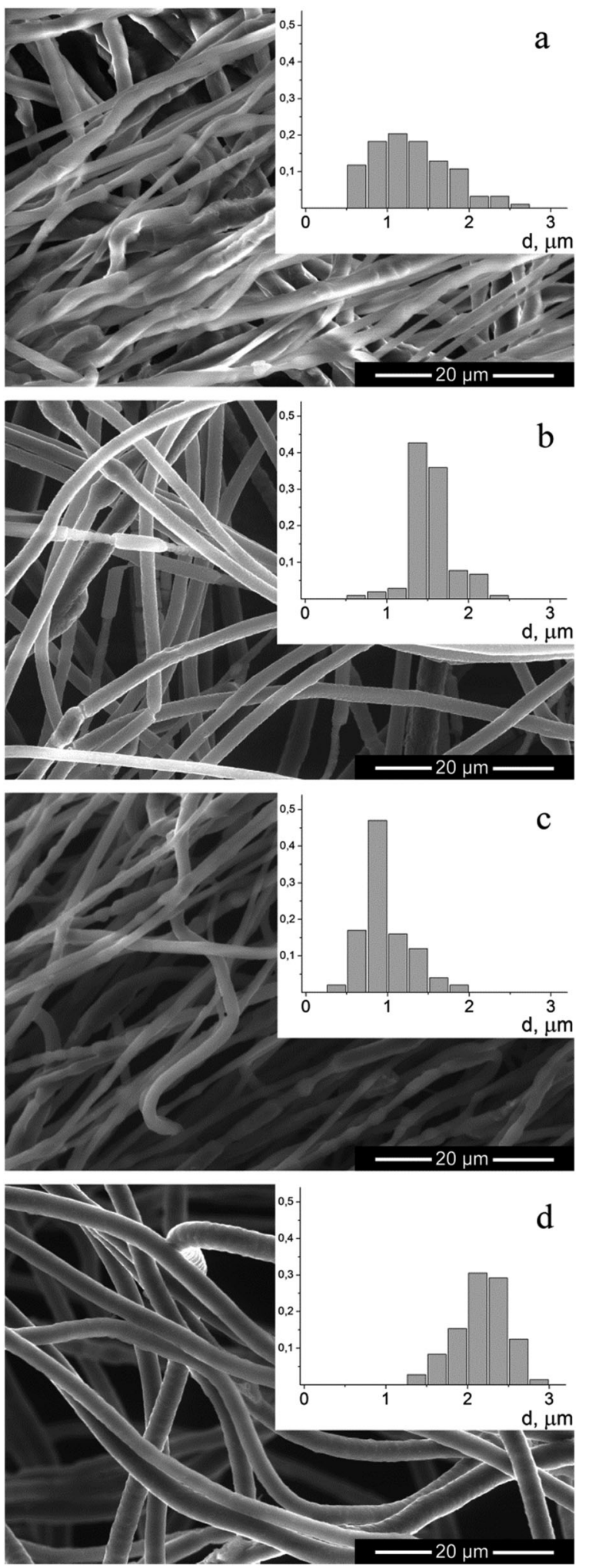

Fig. 8 Micrographs and histograms of fibers, produced from: (a) PEO; (b) PEO + PANI-100 1:1; (c) PEO + PANI-10 1: 1; (d) PEO + PANI-10 2:1.

circular. The average fiber diameter in the case of PEO solution spinning was $1.3 \mu \mathrm{m}$, which is a typical result for this polymer. ${ }^{23}$ The distribution of fiber diameters narrows when spinning from solutions of mixtures of PANI-PEO. When spinning the mixtures of PANI-PEO in the ratio $1: 1$ we obtained fibers with an average diameter of $1.0 \mu \mathrm{m}$ (for PANI-10) and $1.5 \mu \mathrm{m}$ (for PANI-100). An increase of the content of PANI-10 in the mixture to $2: 1$ leads to an increase in the average fiber diameter to $2.2 \mu \mathrm{m}$. When the ratio of PANI: PEO increases to $3: 1$, fibers in the resulting material are not completely dried, which leads to the formation of fibrous-film morphology samples.

FTIR spectra of the native PEO and nonwoven materials are shown in Fig. 8. Spectra of native PEO and nonwoven materials contain all characteristic bands of those polymer: ${ }^{24}$ antisymmetric $\left(2947 \mathrm{~cm}^{-1}\right)$ and symmetric $\left(2884 \mathrm{~cm}^{-1}\right.$ and shoulder at $2865 \mathrm{~cm}^{-1}$ ) $\mathrm{C}-\mathrm{H}$ stretching vibrations in $\mathrm{CH}_{2}$ groups; $\mathrm{C}-\mathrm{H}$ bending vibrations in $\mathrm{CH}_{2}$ groups: scissoring (1467 and $1455 \mathrm{~cm}^{-1}$ ), wagging $\left(1413,1360\right.$ and $\left.1342 \mathrm{~cm}^{-1}\right)$, twisting (1280, 1241 and $1235 \mathrm{~cm}^{-1}$ ), rocking (962, 948 and 843 $\mathrm{cm}^{-1}$ ); $\mathrm{C}-\mathrm{O}$ and $\mathrm{C}-\mathrm{C}$ stretching vibrations $(1147,1100$ and $1061 \mathrm{~cm}^{-1}$ ).

FTIR spectra of nonwoven materials based on PEO and PANI/CSA mixtures also contain peaks which can be assigned to PANI (3062 and $3244 \mathrm{~cm}^{-1}, 1607 \mathrm{~cm}^{-1}$ and $1581 \mathrm{~cm}^{-1}$, bending vibrations of benzenoid and chinoid rings; $1373 \mathrm{~cm}^{-1}$ and $1304 \mathrm{~cm}^{-1}, \mathrm{C}=\mathrm{N}$ stretching vibration) and CSA $\left(1741 \mathrm{~cm}^{-1}\right.$, $\mathrm{C}=\mathrm{O}$ stretching vibration; $1040 \mathrm{~cm}^{-1}$, symmetric vibration of sulfonate groups). The intensity of these bands increases with increasing content of PANI in the mixtures.

Specific conductivity of the fibers with different PANI contents is shown in Fig. 9. The conductivity drastically increases with increasing PANI content by more than $20 \%$ and reaches a plateau at about $30 \%$ having the value about the pure PANI (emeraldine salt) conductivity $\left(4 \times 10^{-3} \mathrm{~S} \mathrm{~cm}^{-1}\right)$. Such dependence of conductivity from a fraction of PANI has percolation nature and notices uniform distribution of PANI in PEO fibers.

Hybrid PANI-PEO fibrous materials contain in each fiber both polymers necessary for an organic memristive device. The creation of stochastic neuromorphic networks based on them requires solving the problem of the reliable conjugation of the

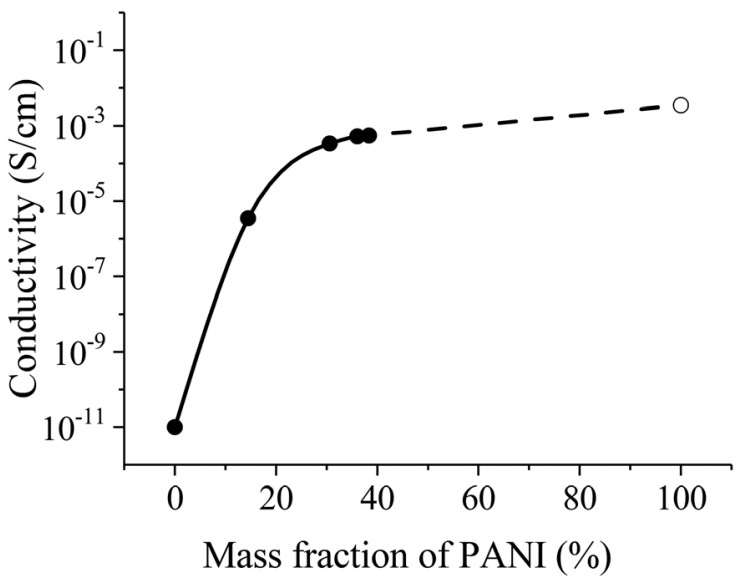

Fig. 9 Conductivity of fibers with different PANI contents. The dashed line extrapolates the experimental data curve to the conductivity of pure PANI (empty circle). 
nonwoven conductive material, a polyelectrolyte matrix and electrodes.

Thus, we created a 3D microfiber nonwoven material promising for creating both separate memristive elements and stochastic neuromorphic networks based on these elements.

\section{Conclusions}

Methods of fabrication of both planar and 3D fibrous PANIbased materials for memristive elements were demonstrated. We have analyzed the influence of PANI molar mass, and natures of solvent and subphase on the formation of structure in Langmuir layers, the thickness and conductivity of LS films. The best conductivity $4.7 \mathrm{~S} \mathrm{~cm}^{-1}$ was reached for the protonated amorphous PANI-100 LS films. The conductivity of partially crystalline films transferred to solid substrates was one to two orders of magnitude lower than the conductivity of amorphous PANI films.

The most conductive films were used for the memristive element fabrication. $I-V$ curves of total current for the elements demonstrate hysteretic behavior with resistive switching. The conductance ratio between high-conductive and low-conductive states of the element at a reading voltage of $+0.3 \mathrm{~V}$ measured as prepared was about 50 and decreased to 9 when measured after 6 days.

Nonwoven materials from PEO and PANI-PEO mixtures with average diameters of 1-2 $\mu \mathrm{m}$ were produced by electrospinning. The conductivity of the PANI-based fibers monotonically increases before the content of $30 \%$ PANI. Then a plateau asymptotic to the pure PANI (emeraldine salt) conductivity $\left(4 \times 10^{-3} \mathrm{~S} \mathrm{~cm}^{-1}\right)$ occurs. The PANI-PEO fibrous material can establish a new promising platform for the realization of stochastic 3D neuromorphic networks, based on organic memristive devices.

\section{Conflicts of interest}

There are no conflicts to declare.

\section{Acknowledgements}

The work was supported in part of planar memristive elements by RSF (project 16-13-00052), in part of nonwoven PANI-based materials by RFBR (projects 16-33-80147 mol_ev_a, 15-29-01324 ofi_m) and President's grant for state support of young Russian candidates of sciences (MK-2757.2017.3). We acknowledge the European Synchrotron Radiation Facility for provision of synchrotron radiation facilities and we would like to thank Konovalov Oleg for assistance in using beamline 10B. Furthermore we acknowledge the resource centers of National Research Center Kurchatov Institute and Rogachev Alexander for assistance in using station "Langmuir".

\section{References}

1 S. H. Jo, T. Chang, I. Ebong, B. B. Bhadviya, P. Mazumder and W. Lu, Nano Lett., 2010, 10, 1297-1301.

2 M. Prezioso, F. Merrikh-Bayat, B. D. Hoskins, G. C. Adam, K. K. Likharev and D. B. Strukov, Nature, 2015, 521, 61-64.

3 V. A. Demin, V. V. Erokhin, A. V. Emelyanov, S. Battistoni, G. Baldi, S. Iannotta, P. K. Kashkarov and M. V. Kovalchuk, Org. Electron., 2015, 25, 16-20.

4 X. Guo, F. Merrikh-Bayat, L. Gao, B. D. Hoskins, F. Alibart, B. Linares-Barranco, L. Theogarajan, C. Teuscher and D. B. Strukov, Front. Neurosci., 2015, 9, 488.

5 C. Evans-Pughe, Eng. Technol., 2010, 5, 30-33.

6 J. Ouyang, Nano Rev., 2010, 1, 5118.

7 D. B. Strukov, G. S. Snider, D. R. Stewart and R. S. Williams, Nature, 2008, 453, 80-83.

8 V. Erokhin, T. Berzina and M. P. Fontana, J. Appl. Phys., 2005, 97, 064501.

9 V. A. Demin, V. V. Erokhin, P. K. Kashkarov and M. V. Kovalchuk, J. Appl. Phys., 2014, 116, 064507.

10 V. Erokhin, T. Berzina, P. Camorani and M. P. Fontana, Colloids Surf., A, 2008, 321, 218-221.

11 T. Berzina, A. Smerieri, G. Ruggeri, M. Bernabo, V. Erokhin and M. P. Fontana, Mater. Sci. Eng., C, 2010, 30, 407-411.

12 T. S. Berzina, K. V. Gorshkov, V. V. Erokhin, V. K. Nevolin and Y. A. Chaplygin, Russ. Microelectron., 2013, 42, 27-32.

13 K. Gorshkov and T. Berzina, BioNanoScience, 2011, 1, 198-201.

14 V. Erokhin, T. Berzina, S. Erokhina and M. P. Fontana, Nano-Net, Springer, Berlin, Heidelberg, 2009, pp. 210-221.

15 V. Erokhin, T. Berzina, A. Smerieri, P. Camorani, S. Erokhina and M. P. Fontana, Nano Commun. Networks, 2010, 1, 108-117.

16 A. Zameshin, I. A. Makhotkin, S. N. Yakunin, R. W. E. van de Kruijs, A. E. Yakshin and F. Bijkerk, J. Appl. Crystallogr., 2016, 49, 1300-1307.

17 M. K. Ram, N. S. Sundaresan and B. D. Malhotra, J. Phys. Chem., 1993, 97, 11580-11582.

18 A. Dhanabalan, R. B. Dabke, N. P. Kumar, S. S. Talwar, S. Major, R. Lal and A. Q. Contractor, Langmuir, 1997, 13, 4395-4400.

19 H. S. Nalwa, Handbook of Organic Conductive Molecules and Polymers, 4 Volumes, Wiley, Chichester, New-York, Weinheim, 1st edn, 1997, vol. 3.

20 W. Lużny, E. J. Samuelsen, D. Djurado and Y. F. Nicolau, Synth. Met., 1997, 90, 19-23.

21 K. Lee, S. Cho, S. H. Park, A. J. Heeger, C. W. Lee and S. H. Lee, Nature, 2006, 441, 65-68.

22 K. Gorshkov and T. Berzina, BioNanoScience, 2011, 1, 198-201.

23 I. D. Norris, M. M. Shaker, F. K. Ko and A. G. MacDiarmid, Synth. Met., 2000, 114, 109-114.

24 F. E. Bailey and J. V. Koleske, Poly(ethylene oxide), Academic Press, 1976. 